\section{SAPping fibrosis}

\section{By Tim Fulmer, Senior Writer}

Researchers from Harvard Medical School and Promedior Inc. have identified a new profibrotic mechanism in the kidney as well as a way to block it with a protein called serum amyloid P. ${ }^{1}$ Promedior is already running a Phase I trial of a recombinant version of the protein to prevent ophthalmic scarring, while preclinical work is ongoing to better understand the role macrophages play in the mechanism and potentially find additional targets.

Serum amyloid P (SAP; APCS) is a naturally circulating protein produced in the liver. Although SAP's name is derived from its initial identification as a minor component of amyloid plaque, it is structurally unrelated to $\beta$-amyloid ( $A \beta)$, the molecule thought to play a role in Alzheimer's disease. Indeed, the precise role of SAP is not entirely clear.

It was known that SAP binds ligands on the surface of pathogens and cells undergoing apoptosis, as well as binding Fc $\gamma$-receptors (FCGRs) expressed on monocytes, macrophages and other innate immune cells.

In 2003, a Rice University team led by Richard Gomer and Darrell Pilling first showed that SAP blocked production of fibrocytes in vitro ${ }^{2}$ and reduced lung fibrosis in rats and mice. ${ }^{3}$ Fibrocytes are monocytederived cells that occur in some fibrotic lesions.

Based on those findings, Gomer and Pilling founded Promedior to focus on the development of monocyte-targeted antifibrotic therapies. SAP was the company's first therapeutic candidate.

One of the first questions Promedior had to answer was how broadly the antifibrotic effects of SAP extended across various diseases and organs. To help answer that question, the biotech has set up collaborations with academic researchers who specialize in specific types of large-organ fibrosis, while the company itself focuses on studying SAP in ophthalmic scarring.

In its most recent publication, Promedior teamed up with Jeremy Duffield, assistant professor of medicine and director of the laboratory of inflammation research at Harvard Medical School and Brigham and Women's Hospital, to study the effects of SAP in the injured kidney.

Duffield and colleagues had previously identified myofibroblasts as important sources of profibrotic collagen in the injured kidney. ${ }^{4}$ Moreover, the presence of macrophages had been shown to be required for the progression of some forms of renal fibrosis. ${ }^{5}$

The open questions were whether SAP targeted any of those cell types and, more importantly, reduced or prevented fibrosis in the injured kidney.

In two different mouse models of kidney fibrosis, intraperitoneal delivery of human SAP significantly reduced the development of fibrosis compared with that in controls $(p<0.05)$.
To get a better handle on the antifibrotic mechanism of SAP, the researchers next looked at its effect on a variety of cell types implicated in driving kidney fibrosis.

In vitro, SAP bound monocytes and macrophages but not collagenproducing fibroblasts, suggesting that its antifibrotic effects were mediated by the immune cells. Additional cell culture studies showed that SAP triggered the release of the anti-inflammatory IL-10 from macrophages and that IL-10 inhibited production of profibrotic collagen by myofibroblasts.

Taken together, the findings suggest that at the site of injury, SAP binds FCGRs on the surface of macrophages, triggering release of IL-10. The cytokine then binds nearby myofibroblasts, inhibiting their production of profibrotic collagen and thus slowing or preventing fibrosis (see Figure 1, "Targeting macrophages in fibrosis").

The results were published in Science Translational Medicine.

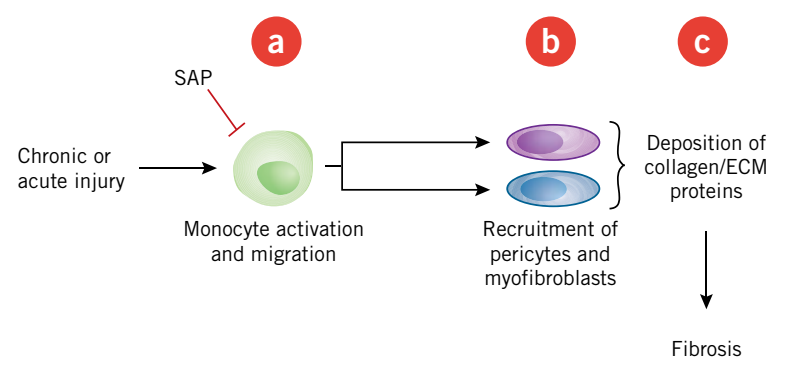

Figure 1. Targeting macrophages in fibrosis. A team of researchers at Harvard Medical School and Promedior Inc. have identified a way to target profibrotic macrophages using an endogenous protein - serum amyloid P (SAP; APCS). ${ }^{1}$

[a] The path to fibrosis starts with chronic or acute injury in solid organs, which leads to the recruitment of multiple immune cells, including macrophages, to the site of injury.

[b] At the injury site, activated macrophages secrete cytokines and chemokines that recruit additional cells. Some of those cells secrete collagen and are profibrotic. The laboratory of corresponding author Jeremy Duffield, assistant professor of medicine at Harvard, has identified two of those profibrotic cell types as myofibroblasts and pericytes.

[c] Chronic or repeat injury triggers the accumulation of increasing numbers of profibrotic cells, which deposit collagen and extracellular matrix (ECM) proteins that contribute to fibrosis and, potentially, organ failure.

The latest findings, published in Science Translational Medicine, show that SAP binds receptors on the surface of macrophages and triggers the release of anti-inflammatory IL-10. In turn, IL-10 binds myofibroblasts to block production of collagen and potentially prevent progression of fibrosis. Promedior has a recombinant form of SAP in Phase I testing to treat ophthalmic scarring and fibrosis associated with some surgical procedures. 


\section{TARGETS \& MECHANISMS}

\section{Going into the clinic}

Although Promedior initially is focused on ophthalmic indications, it ultimately expects to expand to large-organ fibrosis, according to SVP of drug discovery Mark Lupher.

"We'll initially focus on treating scarring associated with certain ophthalmic surgical procedures because ocular imaging techniques provide near-histological resolution of the scarring process and are relatively noninvasive compared with the repeat biopsies that are often needed to show an antifibrotic effect in large organs over time," Lupher told SciBX.

Promedior's recombinant human SAP, PRM-151, started a Phase I trial in healthy volunteers last quarter. Once it has established safety and efficacy in ophthalmic indications, Lupher said, the company hopes to find partners for trials in large-organ fibrosis.

Lupher also told SciBX that Promedior plans to continue working with Duffield. Part of that collaboration could include looking at models of chronic and established renal fibrosis.

Scott Friedman, professor of medicine and chief of the Division of Liver Diseases at the Mount Sinai School of Medicine, said the work in models of acute fibrosis described in Science Translational Medicine "provide solid proof of concept for SAP as an antifibrotic. It's now important to look at SAP in preclinical models of established kidney fibrosis, which more accurately reflect the treatment situation when a patient presents with fibrosis. It remains to be seen whether SAP can block progression of established fibrosis and perhaps at least partially reverse it."

Friedman, who also is president of the American Association for the Study of Liver Diseases, wanted to see evidence that long-term SAP therapy for chronic fibrosis was not immunosuppressive. "Given that SAP is targeting macrophages, there's the possibility that long-term systemic therapy could alter macrophage function and impair the innate immune response, putting the patient at risk of opportunistic infection," he said.

Duffield thinks the innate immune system will not be affected by SAP. "In vitro studies showed that SAP does not inhibit leukocyte activation in response to bacterial cell wall components such as lipopolysaccharide," suggesting that in vivo the compound would not impair the host immune response to pathogens, he said.

Duffield said his next steps include working out the mechanism by which SAP binds FCGRs on macrophages and triggers the release of IL-10.

"A better understanding of the macrophage signaling pathways that mediate the antifibrotic effects of SAP could eventually lead to additional therapeutic targets," he noted.

"Based on our paper's findings, agonists of the activating Fc $\gamma$-receptors and the IL-10 receptor would all be predicted to have a similar antifibrotic effect as administering SAP. Future biochemical characterization of the mechanism we've identified might even eventually lead to small molecule therapeutics that do what SAP does," said Duffield.

Promedior holds multiple patents on the composition of matter and use of SAP to treat fibrotic disorders, including kidney fibrosis.

Fulmer, T. SciBX 2(46); doi:10.1038/scibx.2009.1684

Published online Dec. 3, 2009

\section{REFERENCES}

1. Castano, A. et al. Sci. Transl. Med.; published online Nov. 4, 2009; doi:10.1126/scitranslmed.3000111

Contact: Jeremy Duffield, Brigham and Women's Hospital, Boston, Mass. e-mail: jduffield@rics.bwh.harvard.edu

2. Pilling, D. et al. J. Immunol. 171, 5537-5546 (2003)

3. Pilling, D. et al. J. Immunol. 179, 4035-4044 (2007)

4. Lin, S. et al. Am. J. Pathol. 173, 1617-1627 (2008)

5. Duffield, J. et al. Am. J. Pathol. 167, 1207-1219 (2005)

\section{COMPANIES AND INSTITUTIONS MENTIONED}

American Association for the Study of Liver Diseases, Alexandria, Va. Brigham and Women's Hospital, Boston, Mass. Harvard Medical School, Boston, Mass.

Mount Sinai School of Medicine, New York, N.Y. Promedior Inc., Malvern, Pa.

Rice University, Houston, Texas 